\title{
Extensive Metastasis of Low Grade Uterine Leiomyosarcoma in A Young Patient - A Rare Case Report
}

\author{
Dr.Suryajit Kumar Singh ${ }^{1}$, Dr. Ajeet Kumar ${ }^{2}$, Dr. Rachna Kumari ${ }^{3}$, \\ Dr. Amit Kumar Sinha ${ }^{4}$, Dr. Deepak Kumar ${ }^{5}$
}

\begin{abstract}
Uterine leiomyosarcoma is a rare malignant tumour of uterine smooth muscle cells which median age of diagnosis is usually the $5^{\text {th }}$ to $6^{\text {th }}$ decade with presenting complains of dysfunctionl uterine bleeding and pelvic pain. Here we report a case of extensive metastasis of uterine leiomyosarcoma in a young female who underwent total abdominal hysterectomy one year ago for complain of DUB and pain in lower abdomen. Recently she complained of severe bilateral lower limb pain and went for MRI of waist which revealed multiple large enhancing soft tissue masses suggestive of metastatic lesion. USG also revealed secondary tumoral deposits in retroperitoneum, and both kidneys. FNAC and core needle biopsy from retroperitoneal mass showed features of malignant mesenchymal tumour which was confirmed as metastatic leiomyosarcoma by immunohistochemistry. We discuss this case because of its rarity, extensive metastasis in young age and also to emphasise the importance to differentiate this tumour from other malignant mesenchymal tumour to which it closely resembles.
\end{abstract}

Keywords: Leiomyosarcoma,Malignant mesenchymal tumour uterus

\section{Introduction}

Uterine leiomyosarcoma is a rare malignant tumour of mesenchymal cells and accounting for $1.3 \%$ of all uterine malignancy and $25 \%$ of malignant mesenchymal tumours of uterus. The mean age of diagnosis of uterine leiomyosarcoma is 54yrs with most common presenting symptoms of dysfunctional uterine bleeding and pelvic pain. Most malignant leiomyosarcomas arise independently and are not associated with benign tumours. Uterine leiomyosarcoma has a high propencity for hematogenous spread usually tends to metastasize within pelvis, lungs, liver, and kidneys and rarely metastasize to regional lymphnode. Grossly ,leiomyosarcoma can be well circumscribed and have a tendency for fresh tumour necrosis, haemorrhage, and cystic degeneration and microscopically characterized by hypercellularity, pleomorphism, atypical mitoses and necrosis.

\section{Case history}

A 33years old female complaining of severe pain in both lower limbs for 3 months underwent for MRI of waist with screening of lumbosacral spine.MRI showed multiple large enhancing soft tissue masses in right gluteal region and left inguinal region(fig- 1) suggestive of metastatic deposits. She had a history of total abdominal hysterectomy with salphingo-oopherectomy one year back for benign uterine leiomyoma which was confirmed as low grade leiomyosarcoma on histopathological examination and immunohistochemistry. Her recent USG finding revealed approx $10 \mathrm{~cm}$ sized hetrogenous hypoechoic mass in the left adenexal/retroperitoneal region and large heterogenous mass in upper and mid pole of both kidneys likely secondary tumoral deposits. X-ray chest PA view showed multiple small nodular opacity involving both lung parenchyma. Further USG guided FNAC and core needle biopsy from retroperitoneal mass was performed. FNAC showed predominantly spindle cells few in small clusters and several scattered diffusely with occasional clusters of atypical ells suggestive of malignant spindle cell tumour. Core needle biopsy showed sheets of cells having moderately pleomorphic hyperchromatic nuclei with vacuolated cytoplasm(fig-2)suggestive of malignant mesnchymal tumour. We also carried out an immunohistochemical study for CD10, desmin, SMA, and caldesmon which stained positive(fig-3 \& 4) on our prepared sections. Biochemical examination showed increased blood urea and serum creatine level. Now patient is under chemotherapy with ifosfamide.

\section{Discussion}

Leiomyosarcoma is an aggressive tumor associated with a high risk of recurrence and death, regardless of stage at presentation. The National Comprehensive Cancer Network (NCCN) clinical practice guidelines for uterine sarcomas recommend hysterectomy as the initial treatment of choice for all medically operable patients with disease limited to the uterus. However, in patients with suspected extrauterine disease spread, additional surgical resection of metastatic disease or systemic chemotherapy is recommended after radiological staging. The most common site of leiomyosarcoma of soft tissue is the retroperitoneum accounting for $50 \%$ of all cases. Histological studies of somatic soft tissue leiomyosarcomas have shown that many of these tumours are 
directly arise from the smooth muscle cells lining blood vessels. Uterine fibroids generally don't develop into malignant leiomyomas but uterine leiomyosarcomas(ULMS) frequently coexist within a fibroid. It is found that approximately $0.5 \%$ of women who have hysterectomies for uterine fibroids have leiomyosarcomas. ULMS metastasizes most frequently to lung, peritoneum, bone, and liver. Local recurrence is associated with peritoneal spread. In contrast to endometrial carcinomas which most commonly metastasize to the lymph nodes, ULMS have a high propensity for hematogeneous spread most commonly to the lungs. It is found that leiomyosarcomas are equally common before and after menupause with peak incidence of 40-60yrs. The factors which predicted the risk of extensive metastases in our study were FIGO stage, presence of serosal involvement and local recurrence.

[2]. Tirumani SH, Ojili V, Shanbhogue AK, Fasih N, Ryan JG, Reinhold C. Current concepts in the imaging of uterine sarcoma. Abdom Imaging. 2013;38:397-411

[3]. Leibsohn S, d'Ablaing G, Mishell Jr DR, et al. Leiomyosarcoma in a series of hysterectomies performed for presumed uterine leiomyomas. Am J Obst Gynecol. 1990;162:968-74

[4]. National Comprehensive Cancer Network. NCCN Clinical Practice Guidelines in Oncology Endometrial Cancer Version 1.2013 [Internet]. Fort Washington, PA: National Comprehensive Cancer Network; 2014 [cited 2014 Aug 20]

[5]. Harry VN, Narayansingh GV, Parkin DE. Uterine leiomyosarcomas: A review of the diagnostic and therapeutic pitfalls. Obstet Gynaecol 2007;9:88-94

[6]. Harlow BL, Weis NS, Lofton S. The epidemiology of sarcoma of the uterus. J Natl Cancer Inst. 1986;76:399

[7]. Golden T, Stout AP. Smooth muscle tumors of the gastrointestinal tract and retroperitoneal tissues. Surg Gynecol Obstet 73:784, 1941

\section{Legend}

Fig. 1 : CEMRI showing multiple large enhancing soft tissue masses in right gluteal region

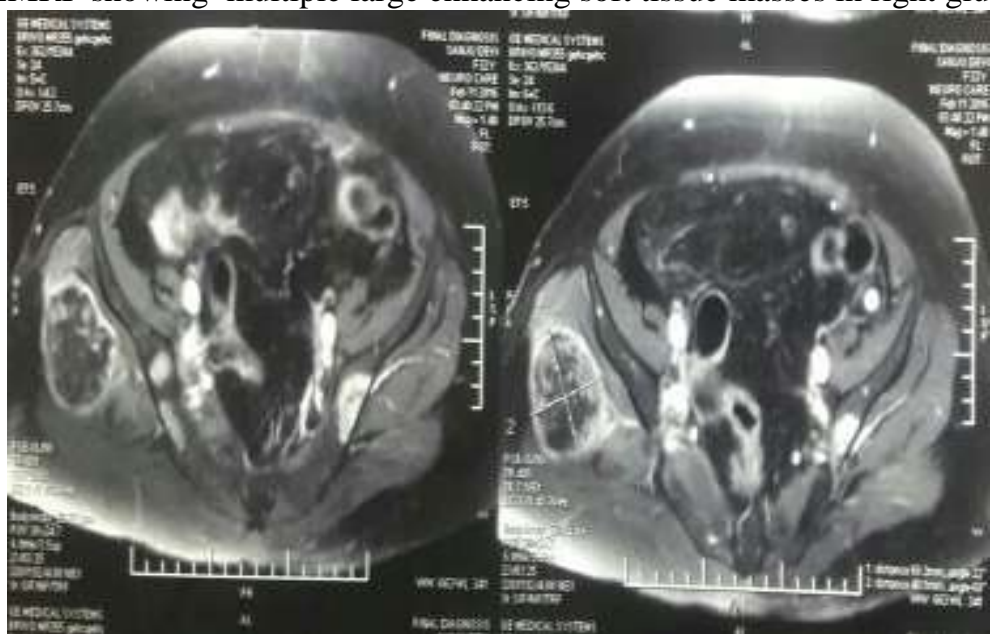

Fig 2: showing sheets of cells having moderately pleomorphic hyperchromatic nuclei with vacuolated cytoplasm

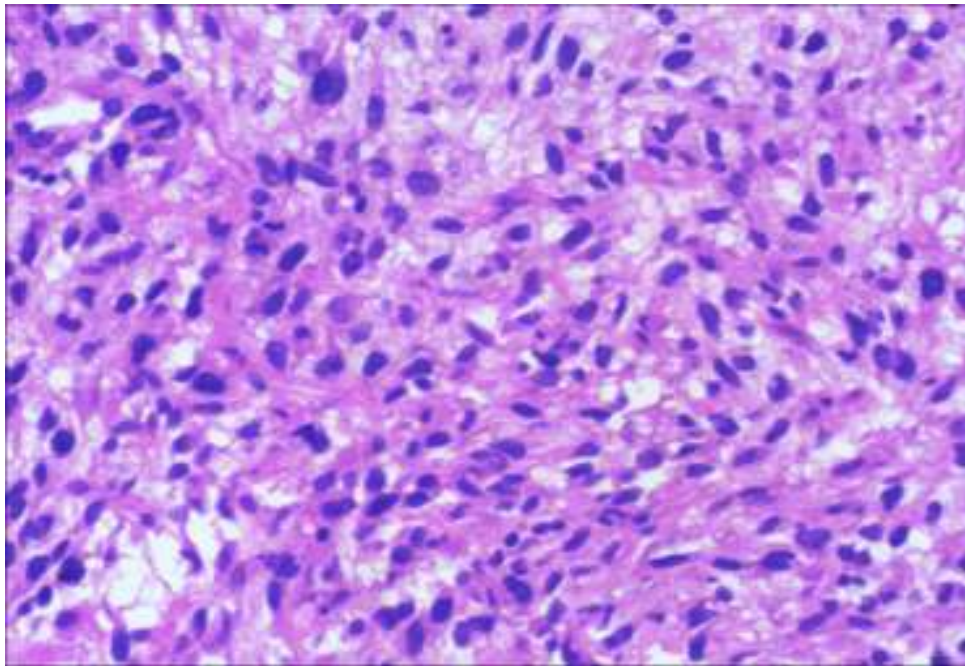

Fig 3:showing tumour cells strongly positive for Desmin 


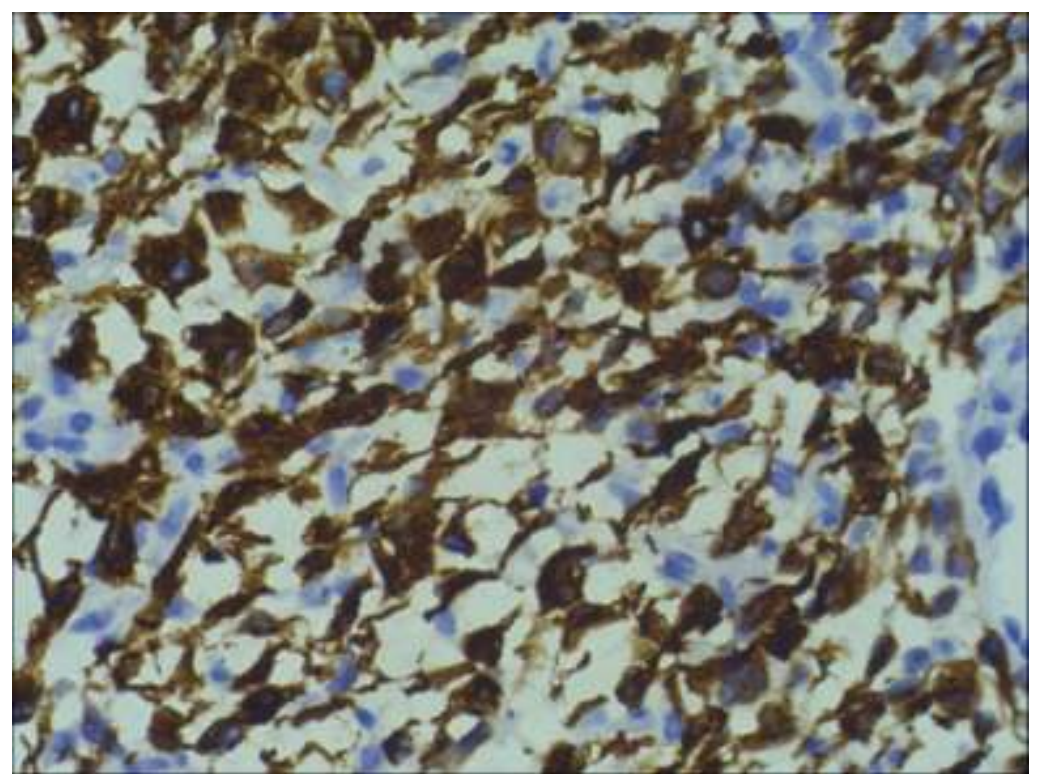

Fig 4 showing SMA positive cells

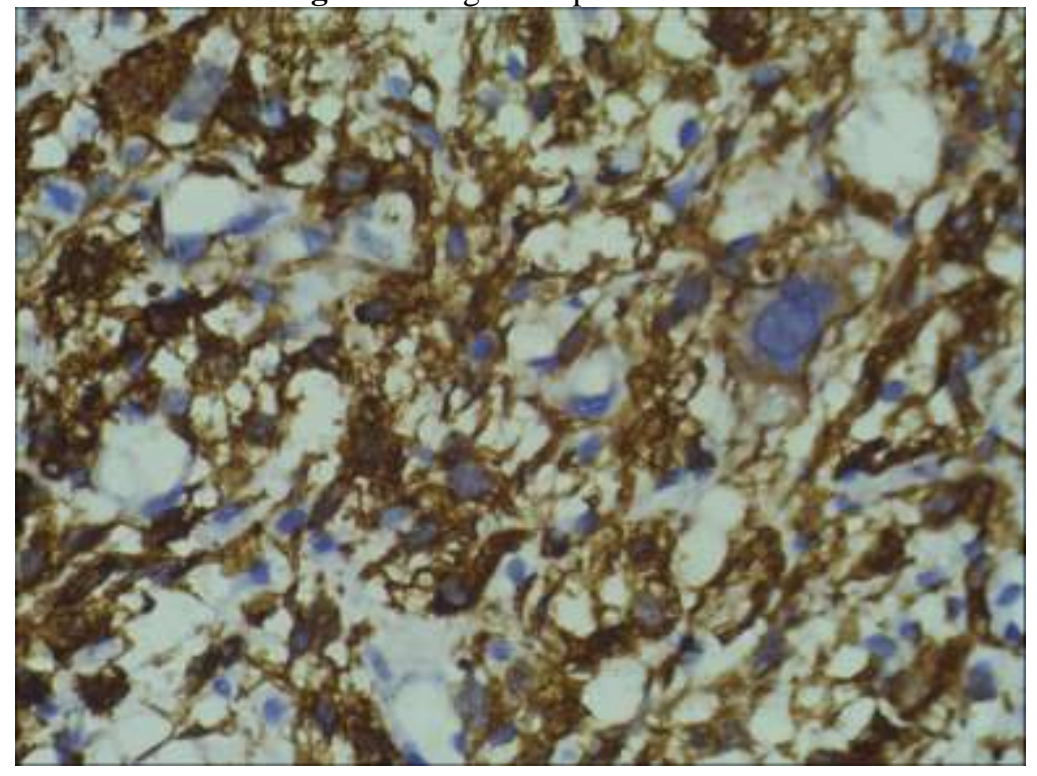

